\title{
Professional player with an in-game ankle injury
}

\section{A. Iqbal ${ }^{1}$ (D) - E. McLoughlin ${ }^{1} \cdot$ R. Botchu ${ }^{1} \cdot$ S. L. James ${ }^{1}$}

Published online: 21 June 2019

(C) ISS 2019

\section{Answer: Isolated calcaneofibular ligament injury}

The MRI images demonstrate an indistinct appearance of the distal half of the CFL with surrounding edema or hemorrhage in keeping with a grade 2 sprain. The ATFL remains intact.

Twenty percent of all sport-related injuries involve the ankle. Inversion injuries account for $85 \%$ of ankle injuries [1]. The LCL is comprised of functionally and morphologically distinct high and low ligaments. The high (syndesmotic) ligaments include the anterior tibiofibular ligament, posterior tibiofibular ligament, and interosseous ligament. The low ankle ligaments include the anterior talofibular ligament (ATFL), calcaneofibular ligament (CFL), and the posterior talofibular ligament (PTFL). While isolated ATFL injury or simultaneous ATFL and CFL injury are common [2], isolated CFL injuries are rarely reported $[3,4]$.

The CFL is an important stabilizer of the lateral ankle and subtalar joints. It arises from the tip of the lateral malleolus and courses postero-inferiorly attaching to the trochlear eminence of the calcaneus. The ligament is typically $6-8 \mathrm{~mm}$ thick and $20 \mathrm{~mm}$ in length and lies deep to the peroneal tendons [5].

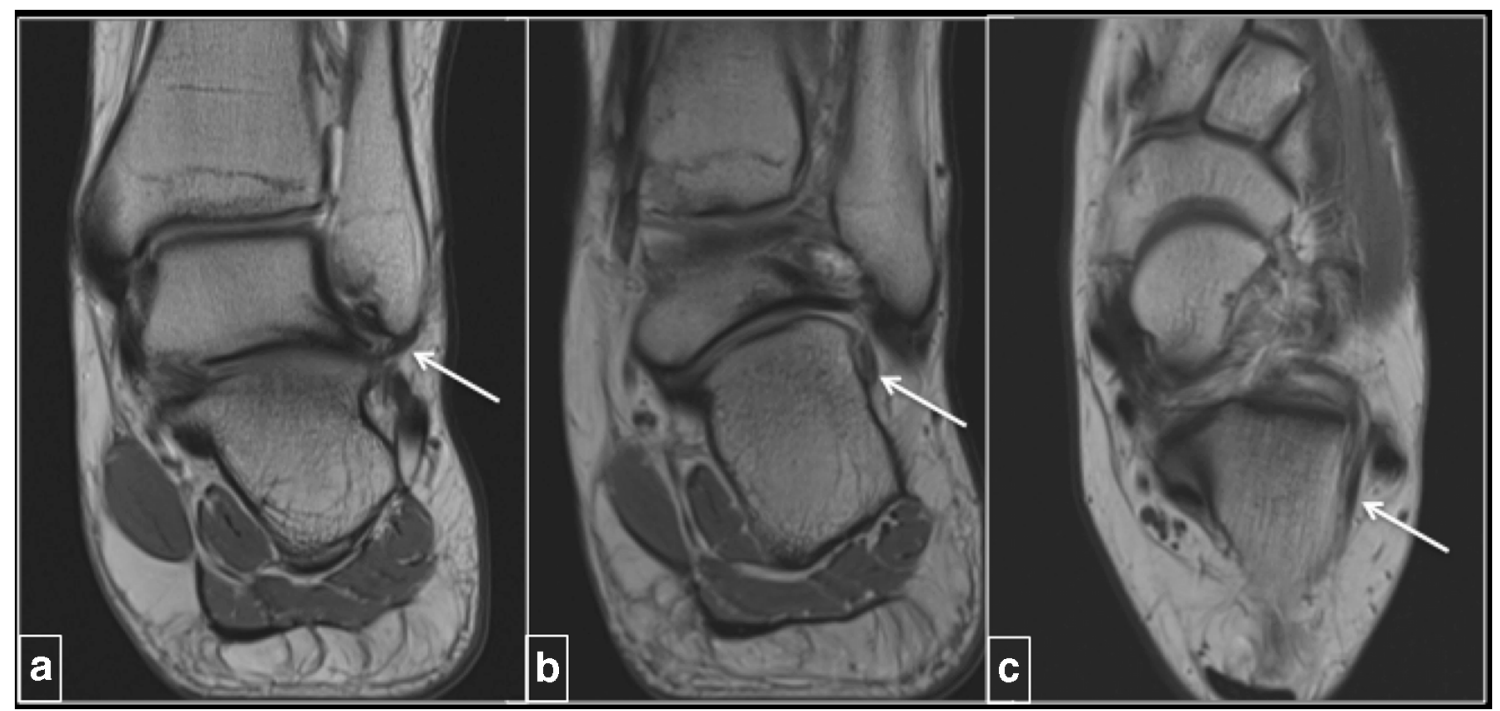

Fig. 1 PD Coronal (a, b) PD axial (c) images demonstrating the proximal, mid, and distal portions of a normal CFL (white arrows)

The case presentation can be found at https://doi.org/10.1007/s00256019-03253-6

A. Iqbal

ai5720@doctors.net.uk

1 Department of Musculoskeletal Radiology, Royal Orthopaedic Hospital NHS Foundation Trust, Bristol Road South, Birmingham, Northfield, UK 


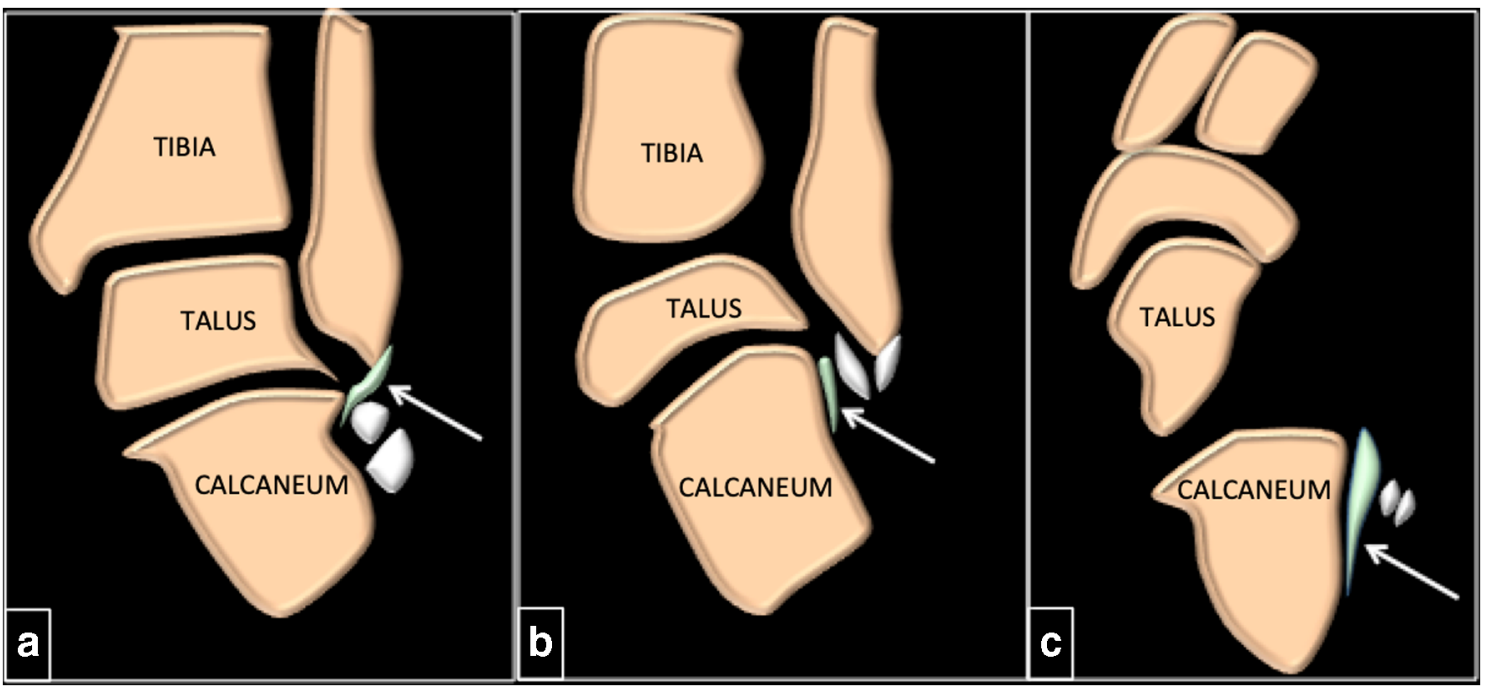

Fig. 2 Coronal $(\mathbf{a}, \mathbf{b})$ and axial (c) illustrated drawings demonstrating the anatomy of the CFL (white arrows) with the peroneal tendons (white) laterally

In vivo studies have shown the ATFL and CFL to have reciprocal functions with the ATFL being responsible for ankle stability in plantarflexion and supination whereas the CFL provides stability during dorsiflexion and inversion [6].

The ATFL is the weakest of the ankle stabilizers and is often the first and most common ligament to injure [4]. This is explained by the gait cycle where the heel makes first contact with the ground in a supinated position followed by plantarflexion of the foot [4]. Inversion of the foot on landing results in ATFL sprain. The CFL is the secondary stabilizer to the ATFL and when it is overstretched, the CFL attempts to reestablish foot alignment. If the forces are too great, the CFL is strained and the PTFL activates. The mechanism for an isolated CFL injury occurs with the ankle in an inverted position with extreme dorsiflexion in order to entirely avoid associated ATFL injury [7].

On MRI, the ATFL and CFL appear as low signal fiber bundles on all sequences. The CFL is best imaged in slight plantar flexion $\left(20^{\circ}\right)$ to reduce the magic angle effect [8]. On axial slices, it appears as a low signal band coursing along the lateral wall of the calcaneus deep to the peroneal tendons. On coronal slices, the ligament is seen as an elongated band extending posteroinferiorly from the tip of the fibula (Figs. 1 and 2).

A range of injuries may affect the ligament including: longitudinal split tear, high-grade partial-thickness tears and complete tears. When injured, the CFL loses its low signal band-like appearance. In high-grade partial tears, the ligament appears thickened and heterogenous with indistinct margins. If completely ruptured the ligament demonstrates a wavy irregular appearance. A number of secondary signs may be encountered including: surrounding soft tissue edema, fluid in the peroneal tendon sheath, and the classic contra-coup bone marrow edema pattern involving the medial joint and deltoid ligament [7]. In chronic injury, the CFL is thickened, scarred, or attenuated.

\section{Conclusions}

Isolated CFL injury is rare and occurs as a result of extreme ankle dorsiflexion and inversion. In the setting of suspected low ankle sprain, MRI assessment requires inspection of the CFL despite intact ATFL.

\section{References}

1. Olds K, Berkowitz MR. Isolated calcaneofibular ligament injuries treated with osteopathic manipulative treatment: a case series. Int J Osteopathic Med. 2012;15:166e172.

2. Al-mohrej OA, Al-Kenani NS. Acute ankle sprain: conservative or surgical approach? Effort Open Rev. 2016;1(2):34-44.

3. Simon JA. The calcaneofibular ligament. MRI Web Clinic- August 2017.

4. Rigby R, Cottom JM, Rozin R. Isolated calcaneofibular ligament injury: a report of two cases. Foot Ankle Surg. 2015;54(3):487-9.

5. Lee M, Hofbauer M. Evaluation and management of lateral ankle injuries. Clin Podiatr Med Surg. 1999;16:659-78.

6. Puffer J. The sprained ankle. Clin Cornerstone. 2001;3:38-49.

7. Asla R, Koz anek M, Wan L, Rubash H, Li G. Function of anterior talofibular and calcaneofibular ligaments during in-vivo motion of the ankle joint complex. J Orthop Surg Res. 2009;4:1-6.

8. Rosenberg ZS, Beltran J, Bencardino JT. From the RSNA refresher courses. Radiological Society of North America. MR imaging of the ankle and foot. Radiographics. 2000;20:S153-79.

Publisher's note Springer Nature remains neutral with regard to jurisdictional claims in published maps and institutional affiliations. 